Pacific Journal of Mathematic 


\title{
EXTENSION OF FUNCTIONS ON FULLY NORMAL SPACES
}

\author{
RICHARD ARENS
}

1. Introduction. Starting from the recent discovery of A. H. Stone that metric spaces are "paracompact" [12] (paracompactness means that every open covering has a refinement only a finite number of whose members meet a suitable neighborhood of each point [5]), J. Dugundji has been able to extend to metric spaces certain techniques in the theory of retracts which were hitherto applicable at most to separable metric spaces [6]. The cornerstone of his method is a theorem (see 2.4, below) according to which a continuous function on a closed set $A$ of a metric space $X$ with values in a convex (= "locally convex") topological linear space $L$ may be extended to the whole space $X$, indeed without enlarging the convex hull of the image. Essentially, the possibility of doing this for a locally separable metric space $X$ is implicitly given by a procedure for the real valued case in [10].

One of the problems to which we address ourselves in this paper is that of determining whether the assumption that $X$ is metric can be reduced to $X$ is merely paracompact. The answer (see 6, below) is no. However, we have fairly general results which imply that if $L$ is metric and complete (and $X$ is paracompact) then the extension is possible ( 4.1 , below). Our proof utilizes a process of extending a pseudo-metric on $A$ to all of $X$, which is ultimately based on a theorem of Hausdorff. We generalize Hausdorff's theorem (3.2 and 3.4) and incidentally show how Dugundji's result enables one to construct a short proof of Hausdorff's theorem.

None of these extension theorems can properly be regarded as a true generalization of Tietze's extension theorem, which deals with mappings on normal spaces with values on the line or in the Hilbert cube, since there exist normal, not fully normal spaces. In order to provide a generalization of Tietze's theorem, we have shown by way of application that the Hilbert cube may be replaced by any compact convex subset of a normed linear space (4.3).

S. Kakutani [10] has introduced the notion of "simultaneous extension regarded as a linear positive operation," in his case of real valued functions on a locally separable metric space $X$ : this means that it is possible so to extend

Received March 10, 1951.

Pacific J. Math. 2 (1952), 11-22 
all continuous functions such that $(\lambda f+g)^{e}=\lambda f^{e}+g^{e}$ and $f^{e} \geq 0$ whenever $f \geq 0$, where the superscribed $e$ indicates the extension. We show that this is possible in the more general case in which $X$ is metric and $L$ convex, the order preserving feature of Kakutani's formulation being naturally reformulated as a nonenlargement of the convex hull of the image (2.6). What is perhaps more surprising is that the "simultaneous extension," while possible in more general cases (4.2), is not possible under as general conditions as those under which the individual extension (as in 4.1) is possible. As a matter of fact, we tie up the notion of simultaneous linear and order relation preserving extension with that of "sweeping" a measure from $X$ onto $A$, and thus show that it is not always possible even for compact Hausdorff spaces $X$ (6.1).

The question arises whether among the simultaneous extensions which preserve linear and order relations, there are not some which preserve quadratic polynomial relations as well. It has already been shown by Yoshizawa just when this is possible: at least when $X$ is compact, $A$ must be a retract of $X$.

We have inserted a section (5) showing that the "simultaneous extension" for real valued functions can be derived from the "individual" extension of a suitable continuous function with values in an infinite dimensional space, as well as from the fundamental Lemma (2.1) directly.

In formulating our results, we shall always speak of "fully normal" [13] rather than "paracompact" spaces, although it is known that these two properties coincide [12]. We do this because we use the full normality as such, using Stone's result only for the metric case.

2. Extension of functions on metric space. One of the main geometric ideas underlying the process of extension involved here is contained in the following construction (cf. $[6,4.3])$.

2.1. Lemma. Let $X$ be a metric space, and $A$ a closed subset of $X$. Then there exists a family $g_{V}$ of continuous real-valued functions defined on $X$, and a similarly indexed family of points $a_{V}$ of A such that

2.2. each $g_{V}$ vanishes on $A$, all but a finite number of the $g_{V}$ vanish in some neighborhood of each point of $X$, the sum $\sum_{V} g_{V}(x)=1$ for all $x$ in $X-A$ and each $g_{V}(x)$ is nonnegative;

2.3. for each $a$ in $A$ and each $V$, if $g_{V}(x)>0$ then $d\left(a, a_{V}\right)<3 d(a, x)(d$ is the metric in $X)$ and $d(a, x)<d\left(a, a_{V}\right)+2 d(x, A)$.

Proof. To each $x$ in $X-A$, assign the open sphere of radius $d(x, A) / 4$. Since $X-A$ is metric it is paracompact [12], so that this covering of $X-A$ has a 
refinement $R$ which is "neighborhood finite" (see [12]). For each $V$ in $R$ there is a point $x$ such that $V$ is contained in the open sphere about $x$ of radius $d(x, A) / 4$; select such a point and call it $x_{V}$. Also select $a_{V}$ in $A$ such that $d\left(a_{V}, x_{V}\right)$ $<(5 / 4) d\left(x_{V}, A\right)$. Let $f_{V}(x)=d(x, X-V)$ for every $x$ in $X$. Since $R$ is neighborhood finite, each $x$ in $X$ has a neighborhood on which all but finitely many $f_{V}$ vanish, and so $s(x)=\sum_{V} \in{ }_{R} f_{V}(x)$ is both finite and continuous. Since $s(x)$ is never 0 , the functions $g_{V}=f_{V} / s$ are continuous. It is easy to see that they provide the kind of "Dieudonné partition" required by 2.2 .

We now turn to 2.3. Suppose $g_{V}(x)>0$. Then $x$ belongs to the open set $V$. From what has been said about $V, x_{V}$, and $a_{V}$ follows

$$
d\left(a_{V}, x\right) \leq d\left(a_{V}, x_{V}\right)+d\left(x_{V}, x\right)<(5 / 4) d\left(x_{V}, A\right)+(1 / 4) d\left(x_{V}, A\right) .
$$

Of course

$$
\begin{aligned}
d\left(x_{V}, A\right) & \leq d\left(x_{V}, a\right) \\
& \leq d\left(x_{V}, x\right)+d(x, a)<(1 / 4) d\left(x_{V}, A\right)+d(x, a),
\end{aligned}
$$

and so (3/4) $d\left(x_{V}, A\right)<d(x, a)$. Thus $d\left(a_{V}, x\right)<(6 / 4) d\left(x_{V}, A\right)<2 d(x, a)$. Thus finally we have half of 2.3 , since

$$
d\left(a, a_{V}\right) \leq d(a, x)+d\left(x, a_{V}\right)<d(a, x)+2 d(x, a)=3 d(a, x) .
$$

For the second half of 2.3 , we note first that $d(x, A)>3 / 4 d\left(x_{V}, A\right)$. On the other hand,

$$
d\left(a_{V}, x\right) \leq d\left(a_{V}, x_{V}\right)+d\left(x_{V}, x\right)<(6 / 4) d\left(x_{V}, A\right),
$$

which is thus less than $2 d(x, A)$. Finally,

$$
d(a, x) \leq d\left(a, a_{V}\right)+d\left(a_{V}, x\right)<d\left(a, a_{V}\right)+2 d(x, A) .
$$

Thus the proof of 2.3 is complete.

Geometrically, the lemma given above says that $X-A$ can be so mapped into the finite dimensional faces of the "formal simplex" with vertices equal to the points of $A$, in such a way that as $x$ tends to a point $a_{0}$ of $A$, the vertices of the carrier of the image of $x$ all tend to $a_{0}$, in the topology of $A$. With this picture in mind, it is easy to imagine how functions on $A$ with values in a convex set $E$, can be extended to all of $X$. The next result $[6,4.1]$ makes this precise. 
2.4. THEOREM. Let $K$ be a convex subset of a convex topological linear space L (cf. [14]). Let $A$ be a closed subset of a metric space X. Let f be continuous on $A$ with values in $K$. Then $f$ may be extended continuously to a function $f^{e}$ defined on $X$ with values in $K$.

Proof. Using the result and notation of 2.1 , we define $f^{e}$ at once by setting $f^{e}(x)=\sum_{V} g_{V}(x) f\left(a_{V}\right)$, for $x$ in $X-A$, and $f^{e}(a)=f(a)$ for $a$ in $A$. There remains only the proof of continuity. Now the topology of $L$ (and thus $K$ ) can be based on neighborhoods of 0 defined by relations $\|k\|<1$ where $\|\cdots\|$ is one of the pseudo-norms of $L$, according to von Neumann's idea (cf. [14]). Select any point $a$ of $A$. There is a positive $r$ such that $d(a, b)<r$ implies $\| f(a)$ $-f(b) \|<1$ for $b$ in $A$. Now suppose $d(a, x)<r / 3$. For those finitely many $V$ for which $g_{V}(x)$ is not 0 , we have $d\left(a, a_{V}\right)<r$, so that

$$
\left\|f^{e}(x)-f(a)\right\| \leq \sum g_{V}(x)\left\|f\left(a_{V}\right)-f(a)\right\|<1 .
$$

This shows the continuity of $f^{e}$ at any point of $A$. At points $x$ of $X-A$ we can find a neighborhood in which only finitely many $g_{V}$ do not vanish, so that $f^{e}$ is continuous there also. The rest of 2.4 is obvious. The second half of 2.3 is not needed for this proof.

The fact that a single formula, so to speak, can be chosen to perform the extension can be expressed in several ways. Suppose $K_{1}$ and $K_{2}$ are convex subsets of two convex topological linear spaces, and let there be an affine mapping $m$ of $K_{1}$ into $K_{2}$. Suppose $f_{1}, f_{2}$ are functions as in 2.4 with values in $K_{1}, K_{2}$ respectively, but satisfying the condition $m\left(f_{1}(a)\right)=f_{2}(a)$ for all $a$ in $A$. If we use the same system $g_{V}, a_{V}$ in extending $f_{2}$ as in extending $f_{1}$ then we surely obtain $m\left(f_{1}^{e}(x)\right)=f_{2}^{e}(x)$ for all $x$ in $X$. We shall abbreviate this by saying that the process of extension when applied to all possible $f$ is consistent, and note the result:

2.5. THEOREM. Each $f$ satisfying the hypothesis of 2.4 with $K$ variablebut $A$ and $X$ constant may be so extended that the entire process is consistent.

Another kind of consistency or simultaneity is expressed as follows.

2.6. THEOREM. Let $K$ be a (linear or possibly merely convex) subset of a convex topological linear space, and let $A$ be a closed subset of the metric space X. Let $F$ be the class of continuous functions on A with values in K. Then each $f$ may be extended by an $f^{e}$ (using 2.4) in such a way that, for $f_{1}, \cdots, f_{n}$ in $F$ and $c_{1}, \cdots, c_{n}$ real numbers (nonnegative with sum 1 when $K$ is merely 
convex), we have

$$
\left(c_{1} f_{1}+\cdots+c_{n} f_{n}\right)^{e}=c_{1} f_{1}^{e}+\cdots+c_{n} f_{n}^{e} .
$$

This result is a generalization of Kakutani's theorem [10] on "simultaneous extension of continuous functions considered as a positive linear operation". The only real advance of 2.6 over Kakutani's theorem is the removal of separability, although Kakutani limits $F$ to the space of bounded real valued continuous functions $C(A)$.

An addendum to 2.4 and 2.6 is of interest:

2.7. Under the conditions of 2.4 or 2.6, if there is an $f$ and a point a of $A$ such that $f$ is constant on a neighborhood (relative to $A$ ) of a, then $f^{e}$ is constant on a neighborhood (relative to X) of $a$.

In fact, suppose $f\left(a^{\prime}\right)$ is constant for $d\left(a, a^{\prime}\right) \leq 3 e$ and $a^{\prime}$ in $A$. Then $f^{e}(x)$ is constant for $d(a, x) \leq e$, since then $d\left(a, a_{V}\right) \leq 3 e$.

3. Extension of pseudo-metrics. Let $X$ be a topological space. Let $s$ be a real-valued function of two variables defined in $X$ such that

$$
s(y, x)=s(x, y) \geq 0, s(x, z) \leq s(x, y)+s(y, z) ;
$$

and such that the set of $x$ such that $s(x, y)<e$ is open for each $e>0$ and $y$. Then $s$ is a pseudo-metric. It falls short of being a metric in that $s\left(x_{n}, y\right) \rightarrow 0$ $[s(x, y)=0]$ does not necessarily imply $x_{n} \rightarrow y(x=y)$. Our first result is in the direction of an extension of a pseudo-metric from a closed set to the whole space.

3.1. Lemma. Let $X$ be a fully normal [13] topological space, and let $q$ be a pseudo-metric defined on a closed subset $A$ of $X$. Then there is a pseudo-metric $s$ defined in all of $X$ such that for $x, y$ in $A$ and $k=4,5, \ldots$, if $s(x, y)<2^{-k}$ then $q(x, y)<2^{-k}$.

Proof. Select a positive integer $n$. Construct an open covering $U$ consisting of those open sets $V$ which intersect $A$ in a set of $q$-diameter less than $2^{-n}$. Using the terminology, notation, and results of [13] we obtain $U \stackrel{*}{>}^{*} U_{1}>^{*} U_{2}>^{*} \ldots$ [13, $\mathrm{V}-7.4]$, and a pseudo-metric $r_{n}$ such that $[13, \mathrm{~V}-7.5$, correcting $\in$ to $\notin]$ $x \notin S\left(y, U_{p}\right)$ implies $r_{n}(x, y)>2^{-(p+2)}$ for $p=1,2, \ldots$. We may also assume $r_{n}(x, y) \leq 1$. We can thus form $r(x, y)=\sum_{n} 2^{-n} r_{n}(x, y)$. This is clearly a pseudo-metric. Suppose $r(x, y)<2^{-k}$, for $k \geq 4$ and $x, y$ in $A$. Then $r_{k-3}(x, y)<$ $2^{-3}$. Hence $x \in S\left(y, U_{1}\right)$, this covering being the one obtained for $n=k-3$. 
(We have omitted an index showing dependence on $n$.) Since $U_{1} \stackrel{*}{<} U$ we obtain $q(x, y)<2^{-k+3}$. Setting $s=2^{-3} r$ gives the required pseudo-metric, completing the proof of 3.1 .

We remind the reader that compact Hausdorff spaces and metric spaces are fully normal [13, V-8.14, VI-4.5].

This lemma is actually all we need in order to extend the results of $\$ 2$ as we shall do later. However, by an application of a theorem of Hausdorff, we can improve 3.1 aesthetically by obtaining a pseudo-metric $s$ which is an extension to $X$ of the original $q$. In fact, rather than refer to Hausdorff's theorem, we first give a new proof since it is an interesting application of 2.1 , is much shorter than Hausdorff's, and shows in passing how a metric space may be isometrically imbedded in its own space of bounded continuous functions (cf. [4, p.187]). The present proof resembles that in [11] more than that in [9]. However, Kuratowski's proof, besides requiring separability, generally does not provide an isometric, but merely topological imbedding (see below, and also [9, p.47]).

3.2. THEOREM. [Hausdorff]. Let $A$ be a closed subset of a metric space $X$, and let $f$ be a continuous mapping of $A$ into another metric space $B$. Then $B$ can be isometrically imbedded in a metric space $Y$ such that $f$ can be continuously extended to $X$ with values in $Y$, such that $f$ is a homeomorphism of $X-A$ with $Y-B$, and such that $B$ is closed in $Y$.

Proof: For any space $S$ let $C(S)$ denote the Banach space of real-valued continuous bounded functions $g$ on $S$, with $\|g\|=\sup _{x} \in S|g(x)|$.

To begin the proof, obtain for $X$ a bounded metric $d$. The metric $r$ in $B$ we must not alter, of course. For $b$ in $B$, let $r_{b}$ denote the function with values $r_{b}\left(b^{\prime}\right)=r\left(b, b^{\prime}\right)$. This function is not necessarily bounded, but $r_{b}-r_{c}$ is bounded (cf. [4, p.187]) and $\left\|r_{b}-r_{c}\right\|=r(b, c)$, where $b, c$ are points of $B$. Select a point $o$ in $A$, to be held constant. The function $\phi$ defined for $a$ in $A$ by $\phi(a)=r_{f(a)}-r_{f(o)}$ evidently maps $A$ into $C(A)$. Indeed, since

$$
\left\|\phi(a)-\phi\left(a^{\prime}\right)\right\|=\left\|r_{f(a)}-r_{f\left(a^{\prime}\right)}\right\|=r\left(f(a), f\left(a^{\prime}\right)\right),
$$

the map $\phi$ is continuous. It may therefore by extended to all of $X$ by 2.4 , and we denote the extension also be $\phi$. Now form $L=C(B) \times R \times C(X)$, where

$$
\|(h, j, k)\|=\max (\|h\|,|j|,\|k\|)
$$

and $R$ is the real number system. For $x, y$ in $X$ dęfine $d_{x}(y)=d(x, y)$ as earlier, and let $d(x)=d(x, A)$. For $x$ in $X$, define $F(x)=\left[\phi(x), d(x), d(x) d_{x}\right]$ in $L$. 
This $F$ is obviously continuous. Define $B_{1}=F(A)$ and $Y=F(X)$, both subsets of $L$. Clearly $B_{1}$ is closed relative to $Y$. Now for each $a$ in $A$ we obtain $f(a)$ on one hand and $F(a)$ on the other. We now show that it sets up an isometry between $B$ and $B_{1}$. In fact,

$$
\left\|F(a)-F\left(a^{\prime}\right)\right\|=\left\|\phi(a)-\phi\left(a^{\prime}\right)\right\|=\left\|r_{f(a)}-r_{f\left(a^{\prime}\right)}\right\|=r\left[f(a), f\left(a^{\prime}\right)\right],
$$

as mentioned earlier. If we identify $B$ with $B_{1}$, then $F$ becomes an extension of $f$, continuous on all of $X$. Suppose $F(x)=F(y)$, where $y$ belongs to $X-A$. Then $d(x)=d(y)>0$; hence $d_{x}=d_{y}$, which means $x=y$. Thus $F$ has an inverse on $Y-B_{1}$. We shall show that it is an homeomorphism. Let $y \in X-A$ and suppose $F(x) \rightarrow F(y)$. Then $d(x) \rightarrow d(y)>0$, and $d(x) d_{x} \rightarrow d(y) d_{y}$. From this we conclude $d_{x} \rightarrow d_{y}$ or $d(x, y)=\left\|d_{x}-d_{y}\right\| \rightarrow 0$. Thus 3.2, Hausdorff's theorem, is proved. It is to be borne in mind that it was not known in 1938 that metric spaces were paracompact.

We go on to establish a refinement of 3.2 also due to Hausdorff.

3.3. THEOREM. If the $f$ in 3.2 is a homeomorphism of $A$ with $B$ then it can be arranged that $F$ also is a homeomorphism.

To establish 3.3 , Hausdorff $[9$, p.46] modifies the construction of $F$. It is an interesting fact that the $F$ we construct automatically satisfies 3.3. The only nontrivial part of the proof of 3.3 is that if $F(x) \rightarrow F(a)$ for $x$ in $X-A$ and $a$ in $A$, then $x \rightarrow a$ in X. Therefore, suppose $F(x) \rightarrow F(a)$. Let

$$
h=g(x)-g(a)=\sum g_{V}(x)\left[r_{f\left(a_{V}\right)}-r_{f(a)}\right],
$$

where the $g_{V}$ and $a_{V}$ are described in 2.1. Now $|h(f(a))| \leq\|h\| \rightarrow 0$. But

$$
h[f(a)]=\sum g_{V}(x) r\left[f\left(a_{V}\right), f(a)\right]
$$

is not less than the least of those $r\left[f\left(a_{V}\right), f(a)\right]$ which appear in this sum, that is, for which $g_{V}(x)$ is not 0 . Denote the $a_{V}$ in question by $a_{W}$, where of course $W$ depends on $x$. Since $r\left(f\left(a_{W}\right), f(a)\right]$ tends to 0 and $f$ is a homeomorphism on $A$, we see that $a_{W} \longrightarrow a$. From 2.3 we obtain $d(a, x)<d\left(a, a_{W}\right)+2 d(x)$, and so $x \rightarrow a$, as desired.

These two results have the following consequence.

3.4. Corollary. Let $A$ be a closed subset of a metric space X. Let $r$ be a pseudo-metric defined on A. Then this pseudo-metric may be extended to all of $X$ in such a way that 
3.41 in $X-A$ it is equivalent to the metric $d$ of $X$;

3.42 in $x \in X-A$ then for some positive $e_{s} r(x, y)<$ e implies $y \in X-A$;

3.43 if $r$ is a metric equivalent to $d$ on $A$, the extension is equivalent to $d$ on all of $X$.

Proof. In $A$, form the equivalence classes for the relation $r(x, y)=0$, and metrize in the obvious way using $r$. Call the resulting space $B$. The natural mapping of $A$ onto $B$ satisfies the hypothesis of 3.2. Let $m$ be the metric in $Y$. Then $m[F(x), F(y)]$ gives the desired extension of $r(x, y)$.

We can now provide the finishing touch to 3.1 .

3.5. THEOREM. Let $X$ be a fully normal topological space, and let $q$ be a pseudo-metric defined on a closed subset of X. Then $q$ can be extended to be a pseudo-metric on $X$.

The proof is based on 3.1 and 3.4 as follows. Using the $s$ of 3.1, partition $X$ into a set $X^{*}$ of equivalence classes according to the relation $s(x, y)=0$, denoting the class containing $x$ by $x^{*}$, and so on. Define $s^{*}\left(x^{*}, y^{*}\right)=s(x, y)$; this is a valid definition, which makes $X^{*}$ a metric space, and the natural mapping of $X$ onto $X^{*}$ is continuous. Let $A^{*}$ be the closure in $X^{*}$ of the image of $A$. The conclusion of 3.1 shows that $q$ may be carried over in unique fashion to $A^{*}$, to form a pseudo-metric $q^{*}$. An appeal to 3.4 extends $q^{*}$ to $X^{*}$, and $q(x, y)=q^{*}\left(x^{*}\right.$, $\left.y^{*}\right)$ provides the desired extension.

Note that we have no use for $3.41-3.43$ in 3.5 because the $s$ was not given to us in advance.

4. Extension of functions on fully normal spaces. In the next result, the metric for $X$ in 2.4 is shifted to $K$.

4.1. TheOREM. Let $A$ be a closed subset of a fully normal space $X$. Let $f$ be continuous on $A$ with values in a complete convex metric subset $K$ of a convex topological linear space $L$. Then $f$ can be continuously extended to $X$ with all values still in $K$.

Proof. In $A$ define the pseudo-metric $q\left(a, a^{\prime}\right)=m\left[f(a), f\left(a^{\prime}\right)\right]$, where $m$ is the metric, and extend $q$ to $X$ by 3.5. Let $A_{0}$ be the set of $x$ such that $q(x, A)$ $=0$. Given $e>0$ and $x$ in $A_{0}$, let $S_{e}$ be the set of $a$ in $A$ such that $q(x, a)<e$. The $f\left(S_{e}\right)$ form a nested system in $K$, and their diameters shrink to 0 . Hence there is just one point, which we call $f(x)$, common to all. This provides an 
extension of $f$ to $A_{0}$. Now partition $X$ into a set $X^{*}$ of equivalence classes under the relation $q(x, y)=0$, denoting the class containing $x$ by $x^{*}$, and so on. Define $q^{*}\left(x^{*}, y^{*}\right)=q(x, y)$; this makes $X^{*}$ into a metric space and the continuous natural image of $X$. In this natural mapping, $A_{0}$ passes onto a closed subset $A^{*}$ of $X^{*}$. The function $f^{*}\left(a^{*}\right)=f(a), a$ in $A_{0}$, is continuous (indeed isometric) on $A^{*}$. It can be extended to all of $X^{*}$, by 2.4. Going back and defining $f(x)=$ $f^{*}\left(x^{*}\right)$, we get an extension of $f$ with the desired properties.

We shall show in 6 that a "simultaneous extension" of the type of 2.6 cannot always be obtained if the hypothesis is merely that of 4.1 for each of the functions involved. However, using the procedure of 4.1 and the result of 2.6 , the reader may prove the following:

4.2. THEOREM. Let $A$ be a closed subset of a fully normal space $X$. Let $F$ be a linear (convex) set of functions each defined on $A$ and with values in a complete metric linear (convex) subset $K$ of a convex topological linear space $L$. Furthermore let there be defined on $A$ a pseudo-metric $q$ such that for each $f$ in $F$ and for each positive $r$ there is a positive $s$ such that $q\left(a, a^{\prime}\right)<s$ implies $m\left[f(a), f\left(a^{\prime}\right)\right]<r_{s}$ where $m$ is the metric in $K$. Then a simultaneous extension (in the sense of 2.6) can be made for all the $f$ in $F$.

None of the preceeding results can properly be claimed to be a generalization of Tietze's extension theorem, since we always require more than normality of $X$. We do not know whether the following is true: if $A$ is a closed subset of a normal space $X$, and $f$ maps $A$ continuously into a bounded closed convex subset $K$ of a Banach space $L$, then $f$ can be continuously extended to $X$ with values in $K$. Of course, in the finite dimensional case of $L$, this result is an easy consequence of the original theorem. In this case, we can replace "bounded" by "compact", and in this form the theorem does admit generalization.

4.3. Тнеовем. Let $A$ be a closed subset of a normal space $X$. Let $K$ be a compact convex subset of a normed linear space L. Let $f$ be a continuous function on A with values in $K$. Then $f$ can be continuously extended to $X$ with values in $K$ (see note added in proof).

Proof: Since $K$ is separable, we can find a countable family $v_{1}, v_{2}, \ldots$ of bounded linear functionals on $L$ such that if $u, u^{\prime}$ belong to $K$ and $u\left(v_{n}\right)=u^{\prime}\left(v_{n}\right)$ for all $n$, then $u=u^{\prime}$. (cf. [2, p.484, "Note"]). We now imbed $K$ in the space (s) of [4]. For $u$ in $K$, define $U(u)_{n}=v_{n}(u)$. This mapping is continuous and one-toone, and hence a homeomorphism. We may therefore forget about the original $L$ and regard $K$ as a compact convex subset of $(s)$. By 2.4, since $(s)$ is metrizable, we can obtain a retraction of $(s)$ on $K$, that is, a continuous $r$ such that $r(u) \in K$ 
for $u$ in $(s)$, and $r(u)=u$ for $u$ in $K$. Let $f_{n}(a)=f(a)_{n}$, the $n$-th coordinate of $f(a)$ in $(s)$. By Tietze's original theorem, this $f_{n}$ may be extended continuously to all of $X$. Defining $f_{0}(x)=\left[f_{1}(x), f_{2}(x), \ldots\right]$ in $(s)$ we obtain a mapping of $X$ into (s). Setting $f^{-}(x)=r\left[f_{0}(x)\right]$ gives the desired extension.

5. Simultaneous extension of real-valued functions. This section merely shows that special cases of 2.6 and 4.2 in which the linear space is the real number system $R$ (or any finite dimensional linear space) can be reduced to 2.4 or 4.1 , respectively, without further inquiry into the method of extension. In other words the possibility of "simultaneous extension" of real-valued functions is a direct consequence of the possibility of a single extension of a function with values in a suitable infinite dimensional space. This sounds quite plausible, but it is perhaps surprising that we must consider conjugate spaces.

Consider first a closed subset $A$ of a metric space $X$, and the spaces $C(A)$ and $C(X)$ of continuous real-valued functions on them. Let $L$ be $C(A)^{-}$, the conjugate space, with the weak topology (see [14], for example). Let $K$ be the set of $\xi$ in $L$ with norm not exceeding 1 and with $\xi \geq 0$ (that is, $\xi(f) \geq 0$ for $f \geq 0$ ). For $a$ in $A$, define $F(a)$ in $K$ by $F(a)(f)=f(a)$. This $F$ is continuous since we are using the weak topology, and $K$ is convex. By 2.4 this $F$ can be extended to $X$. For $f$ in $C(A)$, define $f^{e}$ by $f^{e}(x)=F(x)(f)$. We leave to the reader the completion of the proof of the following:

5.1. THEOREM. The operation $f \rightarrow f^{e}$ is a linear, isometric, nonnegative transformation of $C(A)$ into $C(X)$, and $f^{e}$ is an extension of $f$.

In the next section we shall show that 5.1 cannot be generalized for nonmetric $X$ even if $X$ is compact. However, the following is true.

5.2. THE OREM. Let $A$ be a closed subset of a fully normal space X. Let $S$ be a separable (in the norm topology) closed linear subspace of $C(A)$. Then there is a linear isometric nonnegative transformation $f \rightarrow f^{e}$ of $S$ into $C(X)$ such that $f^{e}$ is an extension of $f$.

The proof is just like that of 5.1, except that we appeal to 4.1. To do this we must observe that since $S$ is separable, $K$ in $S^{-}$with the weak topology is metrizable (as is well known), for example with the metric

$$
m(\xi, \eta)=\sum 2^{-n}\left|(\xi-\eta)\left(f_{n}\right)\right|
$$

where the $f_{n}$ are dense in the unit ball of $S$; and that $K$ is compact (AlaogluBourbaki [1]) and thus complete. 
6. Applications to measure in topological spaces. Let $X$ be a topological space with a measure, and let $A$ be a subset such that every function of a fixed linear set $F$ of real-valued functions on $A$ can be extended to a summable function on $X$ by a positive linear operation $P$. By defining $J(f)=\int P(f)(x) m(d x)$ for $f$ in $F$, we obtain a functional which may sometimes be represented by an integral (cf. $[2,3]$ or any of the references given there). When this is true, one obtains a measure $m^{\prime}$ on $A$ which is generally not the mere restriction $\left[m^{\prime}(E)\right.$ $=m(E)$ for $E \in A]$ of $m$ to $A$.

Unfortunately we have not been able to apply this process to any situation to obtain measures in $A$ of a class not more easily obtainable by other methods. This is because of the requirement of the existence of a pseudo-metric in 4.2 with the stated properties, or of the separability of $S$ in 5.2. The interest of the present section lies mainly in the fact that it is shown that one cannot avoid limitations of this sort. For this purpose we present only one of a variety of theorems, and then show why it cannot be generalized.

6.1. The orem. Let $X$ be a fully normal Hausdorff space and let $m$ be a finite Baire measure [8] such that $m(V)=0$ for an open $V$ only if $V$ is void. Let $A$ be a compact subset of X. Let $S$ be a separable subset of $C(A)$. Then there exists a strongly regular measure $m^{\prime}$ in $A$ such that all functions in $S$ are measurable and if $f \in S$ and $f \geq 0, f \neq 0$ then

$$
\int_{A} f(a) m^{\prime}(d a)>0
$$

Proof. Let $Q$ be the normed linear algebra generated by $S$ and 1 . By $[3,4.4]$ we can obtain a measure as described such that

$$
\int_{A} f(a) m^{\prime}(d a)=\int_{X} f^{e}(x) m(d x)
$$

The point to observe is that if $f \geq 0, f \neq 0$, then the same thing is true for $f^{e}$, and thus the right integral is positive.

Why can we not ignore the separability of $S$ in 6.1? Let $A_{0}$ by any uncountable discrete set. By adding a "point at infinity" we obtain a compact space $A$. This space $A$ can be imbedded in a cartesian product $X$ of unit intervals. The obvious product measure $[8, \mathrm{p} .158(2)]$ has the properties needed for 6.1 . Let $S$ $=C(A)$, and, forgetting that $S$ is not separable, apply 6.1. The resulting measure would make every one of the points $A_{0}$ have nonzero measure, and so $A$ itself would not be measurable. This shows why the separability of $S$ in 6.1 cannot be ignored; and it also shows that one cannot ignore the pseudo-metric $q$ in 4.2 or the separability of $S$ in 5.2 . 
Added in proof: We have recently found, and shall soon publish, a stronger form of 4.3 , namely in which "compact" is replaced by "separable".

\section{REFERENCES}

1. L. Alaoglu, Weak topologies of normed linear spaces, Ann. Math. 41 (1940), 252-267.

2. R. Arens, A topology for spaces of transformations, Ann. Math. 47 (1946), 480-495.

3. R. Arens, Representation of functionals by integrals, Duke Math. 17 (1950) 499-506.

4. S. Banch, Théorie des Opérations Linéaires, Warsaw, 1932.

5. J. Dieudonné, Une généralization des espaces compacts, J. Math. Pure Appl. 23 (1944), 65-76.

6. J. Dugundji, A generalization of Tietze's theorem, Pacific J. Math. 1 (1951), $353-367$.

7. I. Gelfand and G. Silov, Über verschiedene Methoden der Einführung der Topologie in die Menge der maximalen Ideale eines normierten Ringes, Rec. Math., N.S. 9 (1941), 25-39.

8. P. R. Halmos, Measure Theory, New York, 1950.

9. F. Hausdorff, Erweiterung einer stetigen Abbildung, Fund. Math., 30 (1938), 40-47.

10. S. Kakutani, Simultaneous extension of continuous functions considered as a positive linear operation, Japan. Math. 17 (1940), 1-4.

11. C. Kuratowski, Remarques sur les transformations continus des espaces metriques, Fund. Math. 30 (1938), 48-49.

121 A. H. Stone, Paracompactness and product spaces, Bull. Amer. Math. Soc. 54 (1948), 969-977. 


\section{EDITORS}

HERBERT BUSEMANN

University of Southern California

Los Angeles 7, California
R. M. ROBINSON

University of California

Berkeley 4, California

E. F. BECKENBACH, Managing Editor

University of California

Los Angeles 24, California

\section{ASSOCIATE EDITORS}

\author{
R. P. DILWORTH \\ HERBERT FEDERER \\ MARSHALL HALL
}

P. R. HALMOS

HEINZ HOPF

R. D. JAMES

\author{
BØRGE JESSEN \\ PAUL LÉVY \\ GEORGE PÓLYA
}

J. J. STOKER
E. G. STRAUS

KÖSAKU YOSIDA

\section{SPONSORS}

UNIVERSITY OF BRITISH COLUMBIA

CALIFORNLA INSTITUTE OF TECHNOLOGY

UNIVERSITY OF CALIFORNIA, BERKELLEY

UNIVERSITY OF CALIFORNIA, DAVIS

UNIVERSITY OF CALIFORNIA, LOS ANGELES

UNIVERSTTY OF CALIFORNIA, SANTA BARBARA

OREGON STATE COLLEGE

UNIVERSITY OF OREGON
UNIVERSETY OF SOUTHERN CALIFORNIA STANFORD UNIVERSITY

WASHINGTON STATE COLLEGE UNIVERSTTY OF WASHINGTON

AMERICAN MATHEMATICAL SOCIETY NATIONAL BUREAU OF STANDARDS, INSTITUTE FOR NUMERICAL ANALYSIS

Vari-Type Composition by

Elaine Barth

Delores Gilbertson

With the cooperation of

E. F. Beckenbach

E. G. Straus

Printed in the United States of America by

Edwards Brothers, Inc., Ann Arbor, Michigan

UNIVERSITY OF CALIFORNIA PRESS * BERKELEY AND LOS ANGELES

COPYRIGHT 1952 BY PACIFIC JOURNAL OF MATHEMATICS 


\section{Pacific Journal of Mathematics}

\section{Vol. 2, No. $1 \quad$ January, 1952}

Tom M. (Mike) Apostol, Theorems on generalized Dedekind sums ........ 1

Tom M. (Mike) Apostol, Addendum to 'On the Lerch zeta function' ........ 10

Richard Arens, Extension of functions on fully normal spaces ........... 11

John E. Maxfield, A short proof of Pillai's theorem on normal numbers.... 23

Charles B. Morrey, Quasi-convexity and the lower semicontinuity of multiple integrals................................ 25

P. M. Pu, Some inequalities in certain nonorientable Riemannian manifolds....................................... 55

Paul V. Reichelderfer, On the barycentric homomorphism in a singular

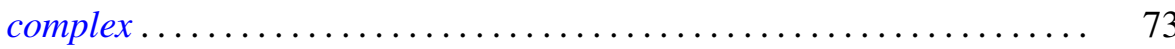

A. H. Stone, Incidence relations in multicoherent spaces. III ........... 99 